\title{
Outcomes of intraoperative versus preoperative ultrasound-guided wire localization of nonpalpable breast lesions
}

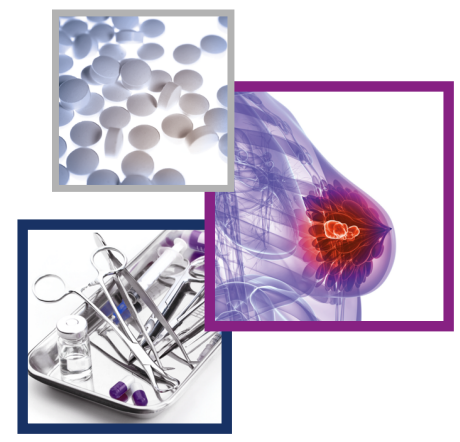

\author{
Primeera Wignarajah*,1 (D), Vasiliki Papalouka² \& Parto Forouhi ${ }^{1}$ (D) \\ ${ }^{1}$ Department of Breast Surgery, Cambridge Breast Unit, Addenbrookes' Hospital, Cambridge University Hospitals Trust, Hills Road, \\ Cambridge, Cambridgeshire, CB2 0QQ, UK \\ 2Department of Radiology, Cambridge Breast Unit, Addenbrookes' Hospital, Cambridge University Hospitals Trust, Hills Road, \\ Cambridge, Cambridgeshire, CB2 0QQ, UK \\ *Author for correspondence: wignarajahp@gmail.com
}

Background: Nonpalpable breast lesions require localization, the gold standard for which is preoperative ultrasound-guided wire localization (PUGWL). Our unit also employs intraoperative ultrasound-guided wire localization (IUGWL). Here we evaluate PUGWL and IUGWL outcomes between 2014 and 2018. Primary outcomes were reoperation rates, complication rates and average specimen weights. Trainee feedback and cost analysis assessed IUGWL viability. Methods: Prospectively recorded data were collected. 511 patients were included (241 PUGWL and 270 IUGWL). Results: Reoperation rates: PUGWL $17.7 \%$ versus IUGWL 13.9\% ( $p=0.28)$. Complication rates: PUGWL 5.8\% versus IUGWL $6.6 \%(p=0.72)$. Average specimen weight: PUGWL 34.2 g versus IUGWL $24.3 \mathrm{~g}$ ( $p<0.0001)$. Trainees needed 15 supervised cases to be IUGWL competent. Performing IUGWL saves $£ 289$ per localization. Conclusion: IUGWL outcomes are comparable to those of PUGWL. IUGWL is cost-effective, patient-friendly and easy to learn and replicate. IUGWL merits wider dissemination and further planned research.

First draft submitted: 11 March 2020; Accepted for publication: 25 November 2020; Published online: 10 May 2021

Keywords: breast cancer • breast conserving surgery • guidewire localization • intraoperative ultrasound - nonpalpable

\section{Background}

In 2017-18 the UK National Health Service Breast Cancer Screening Programme screened 2.14 million women, detecting over 18,000 breast cancers. $40.1 \%$ of these patients had small invasive cancers [1].

Our tertiary breast cancer unit treats around 500 cancers annually, $40 \%$ of which are screen detected. The vast majority of screen-detected lesions are nonpalpable lesions (NPL). Patients with partial or complete response to neoadjuvant therapy add to the number of NPLs treated.

NPLs require localization for breast-conserving surgery (BCS). Traditionally this is performed preoperatively by a Radiologist, using image guidance placing a guidewire into the lesion under local anesthetic. A mammogram then confirms guidewire placement.

Most lesions are ultrasound visible and this is the most commonly used imaging technique for guidewire localization [2-4]; it is faster than stereotactic guidance, and easily operated. Calcification and disease not sonographically visualized can be made localizable using a device often referred to as a marker clip [5,6]. The marker clip is placed in the center of the lesion at diagnostic biopsy. Further mammographic imaging ascertains if it is correctly positioned prior to localization. Patients undergoing neoadjuvant treatment have one or more marker clips deployed prior to commencing systemic treatment. The position of the marker clip, the size of the lesion and the relationship between the two are reassessed with ultrasound prior to surgery [7].

Patients having BCS for a NPL are kept busy on the day of surgery with trips to nuclear medicine, radiology and finally the operating theatre. They also potentially have two invasive procedures: ultrasound-guided localization

Future Medicine 
with subsequent mammography and nuclear medicine injection for sentinel lymph node biopsy. This adds to the patient's anxiety, stress and physical discomfort.

A preoperative ultrasound-guided wire localization (PUGWL) is a costly technique requiring complex organizational arrangements around scarce resources in radiology departments. From a surgeon's perspective, guidewires carry the risk of displacement and migration [8-11] and may take a tortuous route through the breast, increasing the difficulty of BCS. Alternative isotope-based techniques for excision of NPLs, such as radio occult lesion localization or radioactive seeds, carry their own organizational difficulties and are increasingly difficult to implement due to regulations surrounding isotope use. Techniques such as magnetic seeds or radiofrequency transponder devices overcome some of these difficulties but require dedicated equipment and are usually priced to match the cost of a preoperative localization. All intraoperative localization techniques require additional training.

Ultrasound devices are used in multiple clinical settings and are increasingly readily available in many operating theatres. Demonstration of a known breast lesion is a basic function of breast ultrasound and can be employed intraoperatively to localize a NPL or marker clip [12-17]. While ultrasound alone can be used to locate a lesion, the addition of a guidewire provides haptic feedback when NPLs are being excised, increasing confidence in excision, saving time and minimizing the extent of resection.

Intraoperative ultrasound-guided wire localization (IUGWL) of NPLs was introduced into our unit after initial evaluation. Here we report on the outcomes of this technique in comparison with the gold standard PUGWL.

\section{Aim}

To evaluate the surgical and oncological outcomes and viability of the IUGWL technique.

\section{Primary outcomes}

Reoperation rates, resection size as assessed by specimen weights and surgical complications in IUGWL and PUGWL.

\section{Secondary outcomes}

To assess the accuracy and precision of localization and specimen excision in both groups using specimen $\mathrm{x}$-rays. To evaluate the learning curve for the IUGWL technique as part of the surgical training program. To evaluate the effect on operating theatre time of the IUGWL technique. To assess the cost-effectiveness of the IUGWL technique.

\section{Patients \& methods}

\section{Subject identification \& data collection}

We used an opportunistic study design using a convenient sample of routinely collected data from patients being managed for breast cancer at our institution.

Data between October 2014 and December 2018 were collected from the electronic records system EPIC ${ }^{\circledR}$. Anonymized data were recorded and analyzed. Proportions were analyzed using Fisher's exact test, and means using Student's $t$ test with Prism software (GraphPad, CA, USA); p-values $<0.05$ were statistically significant.

Consecutive cases between October 2014 and December 2018 were included in the study if they fulfilled all the following inclusion and exclusion criteria.

\section{Inclusion criteria}

Patients with documented NPL.

Patients with core biopsy-confirmed invasive cancer or high grade ductal carcinoma in situ treated with BCS.

Patients with NPL and a sonographically visible lesion or marker clip that could be localized using ultrasound guidance.

Patients who underwent PUGWL or IUGWL for BCS.

Patients who had an intraoperative specimen radiograph taken.

\section{Exclusion criteria}

Patients undergoing diagnostic excision biopsies for benign or indeterminate lesions.

Patients undergoing therapeutic mammoplasties as BCS using either PUGWL or IUGWL.

Patients who underwent a combination of PUGWL and IUGWL for BCS.

Patients who underwent stereotactic guided wire localizations for NPL. 


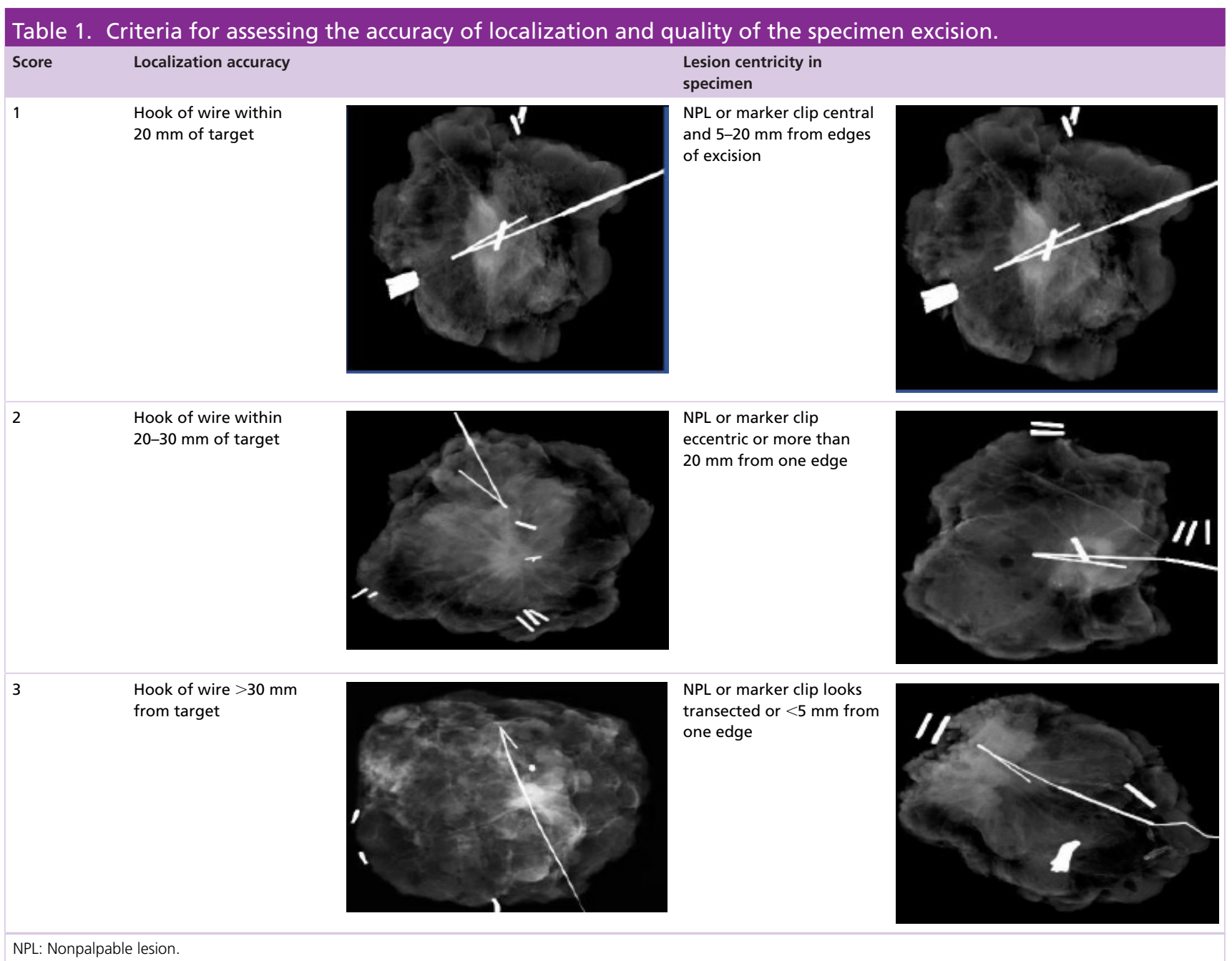

\section{Primary outcomes}

Primary outcomes were reoperation rates, specimen weights and surgical complications.

Reoperation rates were established using the number of patients in each group who either underwent re-excision or proceeded to mastectomy following BSC with positive margins.

Total specimen weight per resection was calculated, including any cavity shaves. Specimen weight is an accurate surrogate for volume [18]. If a patient had more than one lesion excised in separate specimens, each specimen was treated as a separate resection.

Surgical complications were any of the following: wound infection, hematoma, seroma, wound dehiscence, skin necrosis requiring further intervention or treatment. Follow-up was 10-48 months. The fully electronic patient records system EPIC ${ }^{\circledR}$ was used to identify complications.

\section{Secondary outcomes}

Accuracy \& precision of localization \& specimen excision

To assess the accuracy and precision of localization and specimen excision, two surgeons and one radiologist individually measured pre-agreed parameters, based on Quality Assurance Guidelines for Surgeons in Breast Cancer Screening [19] for anonymized specimen x-rays, and assigned a score as set out in Table 1 . The closer to 1 the score, the more accurate the localization and the more centralized the lesion in the excision. Patients with more than one lesion had each lesion scored separately. A proportion of sequential patients were included in this part of the study, excluding those with no identifiable lesion or no guidewire on the specimen x-ray. 


\section{Viability of IUGWL in training surgeons}

To evaluate the learning curve for IUGWL, three senior breast trainees were given one-to-one training by a consultant surgeon. Once deemed competent, the trainees performed IUGWL independently. Training was evaluated with a questionnaire.

\section{Evaluating the effect of IUGWL on operating theatre time}

The electronic records system is updated in real time in the operating theatre. Events are recorded as: time into theatre, anesthetic start time, anesthetic ready time, knife to skin, operation finish time and time out of theatre. Assessment of surgical time was confined to patients undergoing excision of a single breast lesion either by PUGWL or with IUGWL. Anesthetic ready time to operation finish time was collected and analyzed.

\section{Assessing the cost-effectiveness of IUGWL}

The costing team in the hospital's finance department was asked to provide the breakdown and total cost of each procedure.

PUGWL method

PUGWLs are performed by a Radiologist on the day of surgery under local anesthetic in the Radiology department of the Breast Unit. The wire is dressed, and a two-view post-localization mammogram performed and reported. This entire process takes a minimum of 25 min per patient.

\section{IUGWL method}

IUGWLs are performed by the surgeon in the operating theatre. Once the patient is under general anesthesia, prepped and draped, the guidewire is inserted under ultrasound guidance. A post-wire mammogram is not required. No additional operating time is allotted for scheduling IUGWL cases.

A Sonosite Edge ${ }^{\circledR}$ (Amsterdam, Netherlands) ultrasound with a 15-6 MHz linear array probe is used to identify the target (either the lesion or a marker clip) in multiple planes. Our unit uses Hydromark ${ }^{\circledR}$ (Cincinnati, OH, USA) as a marker clip. We initially used a hook guidewire, but recently we have changed to an anchor guidewire which can be introduced one-handed: the Tuloc Premium, Somatex ${ }^{\circledR}$ (Berlin, Germany). An intraoperative specimen radiograph is taken to assess margins.

\section{Results}

511 patients were included in this study. 241 patients had PUGWL for 258 lesions, and 270 patients had IUGWL for 280 lesions.

\section{Patient \& tumor characteristics}

All patients were female. The median age for PUGWL was 63 years (range: 29-86) and median age for IUGWL was 60 years (range: $21-86$ ). $12 \%$ of patients in the PUGWL group were current smokers, compared with $9 \%$ in the IUGWL group. $69 \%$ of patients in the PUGWL group and $68 \%$ in the IUGWL group had recorded comorbidities. 97\% of PUGWL patients and 96\% of IUGWL patients were from a white European ethnic background. There was no significant different between PUGWL and IUGWL with regards to mean size of each index lesion (mm) targeted, patients with sonographic masses or type of disease (Table 2).

\section{Primary outcomes}

Primary outcomes for all cases included in this study are shown in Table 3.

Analysis of patients treated with primary surgery

Table 4 shows primary outcomes and tumour characteristics of patients treated with primary surgery.

\section{Analysis of patients treated with neoadjuvant therapies}

Table 5 shows the primary outcomes and tumour characteristics for patients who underwent neoadjuvant treatment. histology of $16 \mathrm{~mm}$, versus $17.5 \mathrm{~mm}$ in the IUGWL group. The re-excision rate in the PUGWL group was 13/31 
Table 2. Tumor characteristics for all patients included in this study.

\begin{tabular}{|c|c|c|c|c|}
\hline & PUGWL & IUGWL & Total & p-value \\
\hline Total number of patients & 241 & 270 & 511 & \\
\hline Number of resections & 248 & 273 & 521 & \\
\hline Patients with multifocal disease & $16 / 241(7 \%)$ & $10 / 270(4 \%)$ & $26 / 511(5 \%)$ & ns \\
\hline Patients with sonographic mass & $191 / 241(79 \%)$ & $213 / 270(79 \%)$ & $404 / 511(79 \%)$ & ns \\
\hline Patients who underwent neoadjuvant therapy & $32 / 241(13 \%)$ & $62 / 270(23 \%)$ & $94 / 511(18 \%)$ & ns \\
\hline Invasive cancer $+/-$ in situ component & $217 / 248(88 \%)$ & $232 / 273(85 \%)$ & $449 / 521(86 \%)$ & ns \\
\hline Carcinoma in situ (high-grade DCIS) & $31 / 248(12 \%)$ & $41 / 273(15 \%)$ & $71 / 521(14 \%)$ & ns \\
\hline Mean lesion size on histology $(\mathrm{mm})$ & $12.1(0-47)$ & $11.8(0-50)$ & & ns \\
\hline Invasive cancer Grade 1, 2, 3, ungradable & $61,116,39,1$ & $49,114,69,0$ & & 0.01 \\
\hline Nodal status positive & $26 / 217$ & $33 / 232$ & & ns \\
\hline
\end{tabular}

Table 3. Primary outcomes of all preoperative versus intraoperative ultrasound-guided wire localization patients.

\begin{tabular}{|c|c|c|c|}
\hline & PUGWL & IUGWL & p-value \\
\hline Mean specimen weight per resection $(\mathrm{g})$ & 34.2 (range: 3.7-155) & 24.3 (range: $3.1-176)$ & $<0.0001$ \\
\hline Complication rate & $14 / 241(5.8 \%)$ & $18 / 270(6.6 \%)$ & 0.72 \\
\hline Reoperation rate & $44 / 248(17.7 \%)$ & $38 / 273(13.9 \%)$ & 0.28 \\
\hline
\end{tabular}

There was a significant difference between the mean specimen weights per resection in each group: the IUGWL group had lower specimen weights. There was no significant difference in the reoperation rate or complication rate.

IUGWL: Intraoperative ultrasound-guided wire localization; PUGWL: Preoperative ultrasound-guided wire localization.

\begin{tabular}{|c|c|c|c|}
\hline & PUGWL & IUGWL & p-value \\
\hline Total number of patients & 209 & 208 & \\
\hline Median age (years) & 65 (range: $29-86$ ) & 61 (range: $37-86$ ) & ns \\
\hline Number of lesions & 224 & 216 & \\
\hline Number of resections & 215 & 211 & \\
\hline Mean specimen weight per resection (g) & 34.2 (range: $4.9-130$ ) & 22.8 (range: $3.1-105.3$ ) & $<0.0001$ \\
\hline Complication rate & $10 / 209(4.9 \%)$ & $12 / 208(5.7 \%)$ & ns \\
\hline Reoperation rate & $41 / 215(19 \%)$ & $35 / 211(16.5 \%)$ & ns \\
\hline Mean lesion size on histology (mm) & 13.1 (range: $1-47$ ) & 12.9 (range: $1.5-40)$ & ns \\
\hline Invasive cancer grade 1, 2, 3 & $61,101,22$ & $48,96,26$ & ns \\
\hline Nodal status positive & $23 / 184$ & $21 / 170$ & ns \\
\hline High grade DCIS & $31 / 215$ & $41 / 211$ & \\
\hline
\end{tabular}

There was a significant difference in the specimen weights between PUGWL and IUGWL patients. IUGWL patients had a smaller average specimen weight. There were no significant differences in any of the other outcomes.

DCIS: Ductal carcinoma in situ; IUGWL: Intraoperative ultrasound-guided wire localization; ns: Not significant; PUGWL: Preoperative ultrasound-guided wire localization.

$(42 \%)$ and in the IUGWL group $7 / 41(17.5 \%)(\mathrm{p}=0.03)$. The average weight of the resection in the PUGWL group was $32.9 \mathrm{~g}$ and in the IUGWL group was $20.5 \mathrm{~g}(\mathrm{p}=0.01)$.

\section{Secondary outcomes}

To assess quality measures, learning curve, cost effectiveness and effect on operating theatre time of IUGWL compared to PUGWL.

Quality measures of localization \& excision

A total of 334 patients (171 PUGWL and 163 IUGWL) were included in this analysis (Table 6). 


\begin{tabular}{|c|c|c|c|}
\hline & PUGWL & IUGWL & p-value \\
\hline Total number of patients & 32 & 62 & \\
\hline Number of lesions & 34 & 64 & \\
\hline Number of resections & 33 & 62 & \\
\hline Median age (years) & 55 (range: 29-69) & 54 (range: $21-77$ ) & \\
\hline Complication rate & $4 / 32(12.5 \%)$ & $6 / 62(9.7 \%)$ & ns \\
\hline Reoperation rate & $3 / 33(9 \%)$ & $3 / 62(4.8 \%)$ & ns \\
\hline Mean size per lesion on histology (mm) & 5.5 (range: $0-27$ ) & 7.9 (range: $0-50$ ) & ns \\
\hline Invasive cancer grade $1,2,3$, unable to grade & $2,13,17,1$ & $1,19,42,0$ & \\
\hline Nodal status positive & $3 / 33$ & $12 / 62$ & \\
\hline
\end{tabular}

\begin{tabular}{|c|c|c|}
\hline Localization accuracy & PUGWL $(n=167)$ & IUGWL $(n=161)$ \\
\hline 1 = High level of accuracy & $140(84 \%)$ & $137(85 \%)$ \\
\hline $2=$ Medium level of accuracy & $18(11 \%)$ & $17(11 \%)$ \\
\hline $3=$ Low level of accuracy & $9(5 \%)$ & $7(4 \%)$ \\
\hline Lesion centricity in specimen & PUGWL $(n=171)$ & IUGWL $(n=163)$ \\
\hline $1=$ Lesion central & $78(45 \%)$ & $89(55 \%)$ \\
\hline 2 = Lesion slightly eccentric & $61(36 \%)$ & $55(34 \%)$ \\
\hline $3=$ Lesion eccentric or transected & $32(19 \%)$ & $19(11 \%)$ \\
\hline
\end{tabular}

\section{IUGWL effect on operating time}

To establish whether using IUGWL has any effect on operating times, 175 patients in each group having excision of a single lesion were identified. Data recorded in real time for anesthetic ready time and operation finish time were collected and analyzed. The PUGWL group had a mean operating time of $85 \mathrm{~min}$, while the IUGWL group had a mean operating time of $78 \mathrm{~min}(\mathrm{p}=0.002)$.

\section{Trainees evaluation of learning curve}

The three senior surgical trainees reported that they were able to perform the IUGWL procedure independently after 15 proctored insertions, taking a maximum of 5 minutes per procedure. There were no misplaced wires or wire-related complications during the training phase or afterward. All three trainees reported that they will incorporate the technique into their consultant practice. They would also recommend the technique to other trainees and feel competent to teach the technique.

\section{Cost-effectiveness of IUGWL}

PUGWL costs $£ 326$ per procedure, including the cost of a post-localization mammogram.

IUGWL adds no operating theatre time. The additional disposables (guidewire plus sterile ultrasound probe cover) cost $£ 37$ per case. This represents a cost saving of $£ 289$ per case, or $£ 80,920$ for the 280 NPLs excised using IUGWL in this study.

The use of IUGWL also frees approximately $25 \mathrm{~min}$ of radiology time per case.

\section{Discussion}

Techniques for dealing with NPLs have been discussed since 1966 [20]. Over time, the National Health Service Breast Cancer Screening Programme and neoadjuvant therapies have established NPLs as a regular feature on operating theatre lists. Preoperative wire localizations became the gold standard for excision of NPLs, with many surgeons adapting to the technique and demonstrating good results [21-24]. The drawbacks are discomfort and stress 
for the patient [25], impact on list planning and breast clinic, pressure on radiology services and potential for wire migration. IUGWL obviates these issues.

Ultrasound was introduced into preoperative wire localizations in the early 1990s [26]. By the turn of the century intraoperative ultrasound was being explored by many. Initially a tool for simple identification of NPLs intraoperatively, its role was extended to assessing resections and cavities to ensure clear margins. Many studies on intraoperative ultrasound without the use of wires show good results with good rates of clear margins. They also testified to the ease of learning breast ultrasound [27-33].

The scope of intraoperative ultrasound has widened with the use of hydrogel marker clips which made NPLs not normally visible on ultrasound sonographically localizable, thus allowing pathology such as high grade DCIS to be managed with IUGWL.

The use of intraoperative ultrasound combined with surgeon-performed wire localization intraoperatively has only been mentioned in a few studies. Previous studies have reported that intraoperative ultrasound localization using a guidewire or marking needle is a safe technique for NPLs, with good outcomes [34-36]. Our study reports outcomes for the largest number of patients (511 compared with the 214, 28 and 32 from the previous similar studies). Two of the three previous studies focused only on NPLs, as does ours. Shin et al. reported a total of 214 patients, including only those with invasive cancer with or without associated DCIS [34]. Our cohort of patients includes those with only invasive cancer, only high-grade ductal carcinoma in situ and mixed disease. Our study shows favorable re-excision rates and specimen weights with the use of IUGWL for patients with high-grade DCIS alone when compared with PUGWL.

Ultrasound can be used to image the target before and after the wound is open without the use of a guidewire. Indeed, some NPLs can be palpable after initial dissection. Many NPLs, however, remain impalpable after initial dissection. This is particularly the case in NPLs marked with a marker clip, which can be lost during surgery. A skin marker alone, however, is not as helpful as using a guidewire [37].

The haptic feedback from the wire helps orientate the surgeon once the wound is open and tissue mobilized, especially in high-volume less dense breasts. The wire assists in centralizing the lesion in the specimen. Repeated ultrasound inside the wound can be time-consuming. The newer wires allow for a one-handed localization technique to deploy and anchor the wire, with a much-reduced risk of accidental dislodgement. The presence of a guidewire can increase confidence in excision, particularly in the event of loss of a marker clip. By placing the guidewire intraoperatively the surgeon is not limited or constrained in their incision or resection as they may be with PUGWL.

Our study used an opportunistic study design aimed at evaluating the safety and applicability of a technical innovation, by comparing it with a conventional technique. Such a design has inherent limitations, and the study is not intended to provide proof of noninferiority. However, the sample is one of the largest for this type of study reported in the literature, and the results for the IUGWL group are highly reassuring and should encourage others to evaluate the technique in other institutions and settings.

The patients in this study were allocated to either IUGWL or PUGWL according to which operating lists they were assigned to and who they saw in clinic. However, the patient groups had similar characteristics and disease profiles. While patients for primary surgery are assigned to the next available lists, neoadjuvant patients are referred to particular surgeons, and this may have resulted in a greater preponderance of patients operated on after neoadjuvant chemotherapy in the IUGWL group (accounting for the greater number of grade 3 cancers in this group). However, when the primary surgery and neoadjuvant groups were analyzed separately (Tables $4 \& 5$ ), the outcomes were similar for each subgroup. All operations in the IUGWL group were carried out by or supervised by one consultant; however, all were treated according to similar protocols, and all decisions regarding treatment were made by a multidisciplinary team.

Smaller excisions correlate with improved quality of life measures [38]. The IUGWL group had statistically significantly lower specimen weights without increased reoperation rates. Despite the time required for intraoperative guidewire insertion, the IUGWL operations took approximately $10 \%$ less time. We suggest that IUGWL affords the surgeon more confidence in their resection and the centricity of the lesion in the specimen and allows the surgeon to complete the resection more easily, as reflected in reduced operating time. The accuracy of localizations and excision, as assessed by specimen radiographs, were equally good between the two groups.

The viability of any new surgical technique depends on the transferability of skills. We assessed the training needs in IUGWL and found that trainees feel competent to perform the procedure independently after 15 supervised 
attempts. There is good concordance in the trainee feedback, and we plan to extend the trainee evaluation of this study.

The Royal College of Radiologists workforce consensus report in 2018 [39] explains the shortfall in radiologists across the UK, with many hospital trusts struggling to fill the gaps. The pressure on radiology departments throughout the UK continues to mount and is projected to get worse over the next 5 years. Solutions like IUGWL have been welcomed in our department, bringing benefits to both the multidisciplinary team and the patients. We estimate that our unit has saved $£ 80,920$ using this technique between October 2014 and December 2018.

During the COVID-19 pandemic, limiting patient and clinician exposure to what is deemed essential has been part of the strategy to prevent the spread of the virus. The use of the IUGWL technique has proven particularly useful for our unit and our patients during this period. Patients post-chemotherapy and others with NPLs have continued to have surgery during the pandemic.

Not only does the use of IUGWL decrease the footfall across the hospital and reduce exposure for patients and staff, but it has also allowed us to continue operating on patients with NPLs off the main hospital site without having to transfer radiological support. It allows flexibility with operating theatre planning, including evening and weekend elective lists, when departments such as radiology are running on an emergency-only schedule. The use of IUGWL for suitable cases has allowed patients in this highly anxious time to have one less trip to hospital, avoiding close interaction with additional personnel (radiologists, radiographers, support staff) and reducing potential exposure to COVID-19 during times of national lockdown. Given the guidance from the Association of Breast Surgeons for the COVID-19 pandemic, many patients were started on primary endocrine treatment and operated on months later, some with good response and palpable lesions becoming NPLs [40]. Again, the benefit of IUGWL gave us a 'safety blanket' to be able to use ultrasound and localize the lesion if required in the operating theatre on a site without breast radiologists.

\section{Conclusion}

Our study supports the assertion that IUGWL performed by a surgeon is a safe and effective technical innovation in dealing with nonpalpable breast lesions. The technique is cost-effective and the skills required are easily transferable. It merits further dissemination and evaluation.

\section{Future perspective}

Nonpalpable lesions are very common in our clinical practice and are expected to increase within the next 5-10 years considering the ever increasing technological advancements in imaging and screening techniques. New imaging modalities have been under investigation for risk stratification screening such as contrast enhanced mammography, automated whole breast ultrasound and abbreviated MRIs, which are proven to have higher diagnostic accuracy and cancer detection rate than the conventional full field digital mammography (FFDM). To establish the ideal technique for localization of NPLs, future studies should focus on a technique that would minimize patient exposure and appointments, prevents or minimize distress and anxiety to the patient. The ideal technique allows flexibility in scheduling, allows the surgeon to feel confident in identifying the position of the nonpalpable lesion, enables the surgeon to excise the lesion with clear margins and conserve as much breast tissue as possible, allows the surgeon the freedom to place their incision and resect the lesion whichever way they think would provide the best outcome. All whilst being cost-effective and easy to learn and implement. There is continuing innovation in localization techniques for nonpalpable breast lesions such as 3D printing, radioactive seeds, magnetic seeds, carbon marking, radiofrequency identified tags, radioguided occult lesion localization, IUGWL, intraoperative ultrasound localization and indocyanine green. The trend is to move away from preoperative localization and likely to be replaced by one of the newer techniques with time and research. This may however be limited by the costeffectiveness, learning curve and ease of implementation of these various techniques in individual units. Individual units are likely to adopt varying techniques. Ultrasound is already an easily learnt and universally available imaging technique and the skills required to use it intraoperatively require minimal training to learn. As different techniques evolve, it is likely we would resort to a transferable technique which can be used in any breast unit regardless of where the initial biopsy took place. In future, surgical trainees would benefit from training in breast ultrasound and IUGWL techniques to aid their surgical practice as it is a cost-effective, efficient and accurate method of localization as demonstrated in our study. 


\section{Background}

- Nonpalpable breast cancers are common and require localization in order to surgically excise them.

- Various techniques for localization are available, the gold standard is preoperative ultrasound-guided wire localization (PUGWL).

- An alternative technique employed by our unit is intraoperative ultrasound-guided wire localization (IUGWL).

- IUGWL has many advantages for the patient, surgeon and the breast unit, including freedom in list scheduling, creative freedom for the surgical incision, confidence for the surgeon in their excision, prevents stress and discomfort for the patient whilst awake. IUGWL frees up resources such as radiology time and saves the patient having further mammograms.

- IUGWL has a learning curve and needs adequate training in intraoperative ultrasound and localization.

Aims

- This study aims to assess the viability of the IUGWL technique.

- Here we assess the primary outcomes; re-operation rate, specimen weights and complication rate, and the secondary outcomes; accuracy and precision of localization and specimen excision, viability of IUGWL in training surgeons, evaluate the effect of IUGWL on operating theatre time and assess the cost-effectiveness of IUGWL.

Patients \& methods

- Patients who underwent either PUGWL or IUGWL from October 2014 to December 2018 were included.

- Patients included were those with a nonpalpable invasive breast cancer or high grade ductal carcinoma in situ (DCIS) with a visible marker clip or lesion on ultrasound who underwent breast conservation surgery.

- Patient's who were excluded were those who had a benign or indeterminate lesion at core biopsy, those who had a combination of PUGWL and IUGWL, those who required a stereotactic guidewire localization and those who had a therapeutic mammoplasty.

- Data was collected from the electronic records system and analyzed using Prism software.

- A cost analysis of each procedure was conducted.

- Three senior surgical trainees evaluated their experience with the procedure and their cases were assessed for complications of localizations.

- To assess the impact of IUGWL on theatre operating time, real time data of anaesthetic ready time and operation finish time for comparable single lesion IUGWL and PUGWL cases were collected and analyzed.

Results

- 511 patients were included in this study. A total of 241 patients had PUGWL for 258 lesions, and 270 patients had IUGWL for 280 lesions.

- Overall there was a significant difference between the mean specimen weights per resection in each group: the IUGWL group had lower specimen weights. There was no significant difference in the reoperation rate or complication rate.

- We analyzed patients who had primary surgery and neoadjuvant treatment separately.

- In those patients who had primary surgery there was a significant difference in the specimen weights between PUGWL and IUGWL patients. IUGWL patients had a smaller average specimen weight. There were no significant differences in any of the other outcomes.

- In those patients who had neoadjuvant treatment there was no significant difference in the average specimen weight, reoperation rate or complication rate between the two groups for patients.

- To assess the accuracy and precision of localization and specimen excision in the PUGWL and IUGWL groups, a total of 334 patients' specimen x-rays (171 PUGWL, 163 IUGWL) were scored using specific criteria by two surgeons and one radiologist.

- IUGWL and PUGWL groups have similar results in both categories used to assess quality of localization and of excision.

- Comparing operating times in patients undergoing PUGWL versus IUGWL we found PUGWL group had a mean operating time of $85 \mathrm{~min}$, while the IUGWL group had a mean operating time of $78 \mathrm{~min}(p=0.002)$.

- Trainees evaluation of learning curve revealed that three senior trainees felt competent performing IUGWL after 15 proctored cases. They reported their intention to employ IUGWL in their consultant practice and felt confident to train others.

- Assessing the costs for each procedure found that using IUGWL saves $£ 289$ per case, or $£ 80,920$ for the 280 NPLs excised using IUGWL in this study. The use of IUGWL also frees approximately $25 \mathrm{~min}$ of radiology time per case.

Discussion

- Localization techniques for NPLs have been discussed since 1966.

- PUGWL is the current gold standard for localization but it has its draw backs and risks of complications.

- The use of intraoperative ultrasound has increased, with many studies exploring this technique.

- The use of intraoperative ultrasound combined with surgeon-performed wire localization intraoperatively has only been mentioned in a few studies.

- Our study focuses on invasive cancer with or without an in situ component as well as high grade DCIS.

Conclusion

- Our study supports the use of IUGWL in NPL. IUGWL is safe, cost effective with comparable re-operation rates and complication rates to PUGWL. 
Author contributions

P Wignarajah: concept, design, acquisition of data, analysis, interpretation of data, write-up of work. V Papalouka: analysis, review of work. P Forouhi: concept, design, analysis, review of work.

Financial \& competing interests disclosure

The authors have no relevant affiliations or financial involvement with any organization or entity with a financial interest in or financial conflict with the subject matter or materials discussed in the manuscript. This includes employment, consultancies, honoraria, stock ownership or options, expert testimony, grants or patents received or pending, or royalties.

No writing assistance was utilized in the production of this manuscript.

Ethical conduct of research

The authors are accountable for all aspects of the work. The authors have ensured the integrity and accuracy of their work. All procedures followed were in accordance with the ethical standards. Informed consent was obtained from all patients for being included in the study.

Open access

This work is licensed under the Attribution-NonCommercial-NoDerivatives 4.0 Unported License. To view a copy of this license, visit http://creativecommons.org/licenses/by-nc-nd/4.0/

\section{References}

1. Screening and Immunisations Team, NHS Digital. Breast Cancer Screening Programme, England 2017-2018. (2019). https://files.digital.nhs.uk/60/77DCCC/breast-screening-programme-eng-2017-18-report.pdf

2. Rissanen TJ, Mäkäräinen HP, Mattila SI et al. Wire localized biopsy of breast lesions: a review of 425 cases found in screening or clinical mammography. Clin. Radiol. 47(1), 14-22 (1993).

3. Rissanen TJ, Mäkäräinen HP, Kiviniemi HO, Suramo II. Ultrasonographically guided wire localization of nonpalpable breast lesions. $J$. Ultrasound Med. 13(3), 183-188 (1994).

4. Davies AH, Cowan A, Jones P, Watkins RM, Teasdale C. Ultrasound localization of screen detected impalpable breast tumours. J. $R$. Coll. Surg. Edinb. 39(6), 353-354 (1994).

5. McMahon MA, James JJ, Cornford EJ, Hamilton LJ, Burrell HC. Does the insertion of a gel-based marker at stereotactic breast biopsy allow subsequent wire localizations to be carried out under ultrasound guidance? Clin. Radiol. 66(9), 840-844 (2011).

6. Burbank F, Forcier N. Tissue marking clip for stereotactic breast biopsy: initial placement accuracy, long-term stability, and usefulness as a guide for wire localization. Radiology 205(2), 407-415 (1997).

7. Dash N, Chafin SH, Johnson RR, Contractor FM. Usefulness of tissue marker clips in patients undergoing neoadjuvant chemotherapy for breast cancer. AJR Am. J. Roentgenol. 173(4), 911-917 (1999).

8. Mituś J, Kołodziejski L, Dyczek S, Wysocki WM, Komorowski AL. Localization wire migrating into the hilum of the lung during wire-guided breast biopsy. Breast J. 10(2), 165-6 (2004).

9. Banitalebi H, Skaane P. Migration of the breast biopsy localization wire to the pulmonary hilus. Acta Radiol. 46(1), 28-31 (2005).

10. Azoury F, Sayad P, Rizk A. Thoracoscopic management of a pericardial migration of a breast biopsy localization wire. Ann. Thorac. Surg. 87(6), 1937-1939 (2009).

11. Seif A, Axelrod H, Nascimento T et al. Migration of guidewire after surgical breast biopsy: an unusual case report. Cardiovasc. Intervent. Radiol. 32(5), 1087-1090 (2009).

12. Harlow SP, Krag DN, Ames SE, Weaver DL. Intraoperative ultrasound localization to guide surgical excision of nonpalpable breast carcinoma. J. Am. Coll. Surg. 189(3), 241-246 (1999).

13. James TA, Harlow S, Sheehey-Jones J et al. Intraoperative ultrasound versus mammographic needle localization for ductal carcinoma in situ. Ann. Surg. Oncol. 16(5), 1164-1169 (2009).

14. Smith LF, Rubio IT, Henry-Tillman R, Korourian S, Klimberg VS. Intraoperative ultrasound-guided breast biopsy. Am. J. Surg. 180(6), 419-423 (2000).

15. Fortunato L, Penteriani R, Farina M, Vitelli CE, Piro FR. Intraoperative ultrasound is an effective and preferable technique to localize non-palpable breast tumors. Eur. J. Surg. Oncol. 34(12), 1289-1292 (2008).

16. Pan $\mathrm{H}, \mathrm{Wu} \mathrm{N}$, Ding $\mathrm{H}$ et al. Intraoperative ultrasound guidance is associated with clear lumpectomy margins for breast cancer: a systematic review and meta-analysis. PLoS ONE 8(9), e74028 (2013).

17. Volders JH, Haloua MH, Krekel NM, Meijer S, van den Tol PM. Current status of ultrasound-guided surgery in the treatment of breast cancer. World J. Clin. Oncol. 7(1), 44-53 (2016).

18. Parmar C, West M, Pathak S, Nelson J, Martin L. Weight versus volume in breast surgery: an observational study. JRSM Short Rep. 2(11), 87 (2011). 
19. Quality Assurance Guidelines for Surgeons in Breast Cancer Screening (4th Edition). Sibbering M, Watkins R, Winstanley J, Patnick J (Eds). NHS Cancer Screening Programmes, Sheffield, UK (2009).

20. Dodd GD, Fry K, Delany W et al. Preoperative localization of occult carcinoma in the breast. TF Nealon, Management of the Patient with Cancer. Saunders Co, PA, USA (1966).

21. Luoma AA, Scudamore CH, Longley JD. Experience with fine-wire localization breast biopsies for nonpalpable breast lesions detected mammographically. Can. J. Surg. 29(5), 315-318 (1986).

22. Symmonds RE, Roberts JW. Management of nonpalpable breast abnormalities. Ann. Surg. 205(5), 520-528 (1987).

23. Miller RS, Adelman RW, Espinosa MH, Dorman SA, Smith DH. The early detection of nonpalpable breast carcinoma with needle localization. Experience with 500 patients in a community hospital. Am. Surg. 58(3), 193-198 (1992).

24. Ahmed M, Douek M. Intra-operative ultrasound versus wire-guided localization in the surgical management of non-palpable breast cancers: systematic review and meta-analysis. Breast Cancer Res. Treat. 140(3), 435-446 (2013).

25. Kelly P, Winslow EH. Needle wire localization for nonpalpable breast lesions: sensations, anxiety levels, and informational needs. Oncol. Nurs. Forum 23(4), 639-645 (1996).

26. Frenna TH, Meyer JE, Sonnenfeld MR. US of breast biopsy specimens. Radiology 190(2), 573 (1994).

27. Esgueva A, Rodríguez-Revuelto R, Espinosa-Bravo M, Salazar JP, Rubio IT. Learning curves in intraoperative ultrasound guided surgery in breast cancer based on complete breast cancer excision and no need for second surgeries. Eur. J. Surg. Oncol. 45(4), 578-583 (2019).

28. Hoffmann J, Marx M, Hengstmann A et al. Ultrasound-assisted tumor surgery in breast cancer - a prospective, randomized, single-center study (MAC 001). Ultraschall Med. 40(3), 326-332 (2019).

29. Arko D, Čas Sikošek N, Kozar N, Sobočan M, Takač I. The value of ultrasound-guided surgery for breast cancer. Eur. J. Obstet. Gynecol. Reprod. Biol. 216, 198-203 (2017).

30. Eggemann H, Costa SD, Ignatov A. Ultrasound-guided versus wire-guided breast-conserving surgery for nonpalpable breast cancer. Clin. Breast Cancer 16(1), e1-6 (2016).

31. Ramos M, Díez JC, Ramos T, Ruano R, Sancho M, González-Orús JM. Intraoperative ultrasound in conservative surgery for non-palpable breast cancer after neoadjuvant chemotherapy. Int. J. Surg. 12(6), 572-577 (2014).

32. Karadeniz Cakmak G, Emre AU, Tascilar O, Bahadir B, Ozkan S. Surgeon performed continuous intraoperative ultrasound guidance decreases re-excisions and mastectomy rates in breast cancer. Breast 33, 23-28 (2017).

33. Ngô C, Pollet AG, Laperrelle J et al. Intraoperative ultrasound localization of nonpalpable breast cancers. Ann. Surg. Oncol. 14(9), 2485-2489 (2007).

34. Shin YD, Choi YJ, Kim DH et al. Comparison of outcomes of surgeon-performed intraoperative ultrasonography-guided wire localization and preoperative wire localization in nonpalpable breast cancer patients undergoing breast-conserving surgery: a retrospective cohort study. Medicine (Baltimore) 96(50), e9340 (2017).

35. Ivanovic NS, Zdravkovic DD, Skuric Z et al. Optimization of breast cancer excision by intraoperative ultrasound and marking needle technique description and feasibility. World J. Surg. Oncol. 13, 153 (2015).

36. Bouton ME, Wilhelmson KL, Komenaka IK. Intraoperative ultrasound can facilitate the wire guided breast procedure for mammographic abnormalities. Am. Surg. 77(5), 640-646 (2011).

37. de Roos MAJ, Welvaart WN, Ong KH. Should we abandon wire-guided localization for nonpalpable breast cancer? A plea for wire-guided localization. Scand. J. Surg. 102(2), 106-109 (2013).

38. Volders JH, Haloua MH, Krekel NMA et al. Intraoperative ultrasound guidance in breast-conserving surgery shows superiority in oncological outcome, long-term cosmetic and patient-reported outcomes: final outcomes of a randomized controlled trial (COBALT). Eur. J. Surg. Oncol. 43(4), 649-657 (2017).

39. Royal College of Radiologists. Clinical radiology UK workforce consensus 2018 report (2019). https://www.rcr.ac.uk/system/files/publication/field_publication_files/clinical-radiology-uk-workforce-census-report-2018.pdf

40. Association of Breast Surgery. Confidential advice for health professionals on breast service provision. (2020).https://associationofbreastsurgery.org.uk/media/252009/abs-statement-150320-v2.pdf 